\title{
Fast and flexible simulation of DNA sequence data
}

\author{
Gary K. Chen, ${ }^{1,2}$ Paul Marjoram, ${ }^{1}$ and Jeffrey D. Wall ${ }^{2,3}$ \\ ${ }^{1}$ Department of Preventive Medicine, University of Southern California, Los Angeles, California 90033, USA; ${ }^{2}$ Institute for Human \\ Genetics and Department of Epidemiology and Biostatistics, University of California, San Francisco, California 94143, USA
}

\begin{abstract}
Simulation of genomic sequences under the coalescent with recombination has conventionally been impractical for regions beyond tens of megabases. This work presents an algorithm, implemented as the program MaCS (Markovian Coalescent Simulator), that can efficiently simulate haplotypes under any arbitrary model of population history. We present several metrics comparing the performance of MaCS with other available simulation programs. Practical usage of MaCS is demonstrated through a comparison of measures of linkage disequilibrium between generated program output and real genotype data from populations considered to be structured.
\end{abstract}

[Supplemental material is available online at www.genome.org. The MaCS source code is freely available at http:// www-hsc.usc.edu/ garykche/.]

With recent advances in DNA microarray technology, it is now feasible to conduct genome-wide association studies (GWAS) for identifying genetic variants that affect a particular phenotype of interest. Despite the vast amount of genotype data already collected, there are still open questions regarding the optimal experimental design of future association studies and the best methods for analyzing large data sets. One important tool that may help answer these questions is computer simulation. For example, a computationally efficient method for simulating whole-genome single nucleotide polymorphism (SNP) data would allow one to compare different mapping methods, or conduct a power study to determine the optimal study design under specific disease or phenotype models.

Several simulation-based methods have utilized existing data to make inferences about additional unobserved data (either additional SNPs or genotypes from additional individuals), including "hide the SNP" studies, bootstrap resampling studies, and more sophisticated methods (Durrant et al. 2004; Stram 2004; Scheet and Stephens 2006). These data-perturbation methods, though appealing in some ways, have several weaknesses. In particular, there is no guarantee that these methods are accurate-patterns of linkage disequilibrium (LD) at longer distances cannot necessarily be extrapolated from patterns of LD at shorter distances (Wall 2004). Also, data-perturbation methods can only be used for populations that already have extensive SNP data. They are necessarily uninformative about the patterns of LD in other populations.

An alternative approach to making inferences about unobserved data involves simulating under a plausible evolutionary model. Of these "model-based" methods, by far the most popular and most useful is the coalescent (Kingman 1982; Hudson 1991). Model-based methods have the advantage of being able to produce (simulated) data that are not dependent on an existing data set. However, any particular model makes assumptions, and the data produced will necessarily be sensitive to these initial assumptions. For humans, this concern may be somewhat mitigated by knowledge of the basic demographic parameters (Reich et al. 2001; Schaffner et al. 2005; Voight et al. 2005; Plagnol and Wall 2006). The coalescent has been generalized to include a wide variety of biologically realistic scenarios, including models

${ }^{3}$ Corresponding author.

E-mail wallj@humgen.ucsf.edu; fax (415) 476-1356.

Article published online before print. Article and publication date are at http:// www.genome.org/cgi/doi/10.1101/gr.083634.108. of population structure, changes in population size, variable recombination rates, and intragenic gene conversion (Hudson 1991, 2002; Wiuf and Hein 2000; Li and Stephens 2003).

Though the coalescent is flexible enough to simulate many different demographic scenarios, it does not scale well to simulating extremely long stretches of DNA (i.e., hundreds of $\mathrm{Mb}$ ) when recombination events are included in the model. In particular, simulating the entire ancestral recombination graph (ARG), which describes all past coalescent and recombination events for a given sample, poses a large computational burden (Griffiths and Marjoram 1996). For example, we estimate that simulating a medium-sized chromosome $(100 \mathrm{Mb})$ in 2000 individuals would take $\sim 2-3$ mo on a single $2-\mathrm{GHz}$ processor and more than $32 \mathrm{~GB}$ of RAM. Though computers are continually getting faster, it is clear that simulation studies (involving many replicates) of whole-genome SNP data using standard coalescent methodology will not be computationally feasible for many years.

In part because of these limitations, two studies proposed a simplification of the standard coalescent, the Sequentially Markov Coalescent (SMC), that is much more computationally efficient (McVean and Cardin 2005; Marjoram and Wall 2006). While the standard coalescent starts at the present time and simulates backward in time, the SMC starts at one end of the sequence and simulates sequentially across its length. Specifically, the SMC starts with a coalescent tree at the left-hand end of the sequence and progressively modifies the tree with recombination events as it moves to the right. Each successive tree modification generates a new tree at the current position, also known as a local tree. In other words, a local tree explains the genealogy for a region that is flanked by two successive recombination events. Computational efficiency is obtained by assuming that the recombination process operates as a Markov process, namely, that modifications to the tree due to recombination happen independent of any previous recombination events. This allows the runtime of the SMC to scale linearly with the length of the sequence being simulated. In contrast, in the standard coalescent, recombination events do not happen independent of each other (Griffiths and Marjoram 1996), and runtimes scale more than quadratically with sequence length (Marjoram and Wall 2006). The increase in speed of the SMC algorithm is obtained at the cost of a slight degree of approximation to the full ARG.

In this work, we describe an intermediate approach that is a compromise between the accuracy of the standard coalescent and the speed of the SMC. Our method, called MaCS (Markovian 
Coalescent Simulator), proceeds from one end of the sequence to the other as do previous methods (Wiuf and Hein 1999; McVean and Cardin 2005; Marjoram and Wall 2006); like the SMC, it models dependencies between recombination events that are physically close to each other but treats recombination events that are far apart as independent. As with the standard coalescent as implemented in the program $m s$ (Hudson 2002), our algorithm models mutation events based on the infinite sites model (Watterson 1975) where no recurrent mutations occur at any given site. MaCS produces simulated data that are virtually identical to data simulated under the standard coalescent, but in much less time and using much less memory. The source code that implements this method, written in $\mathrm{C}++$, is freely available to the research community at http://www-hsc.usc.edu/ garykche/. This program is as flexible as the widely used coalescent simulator $m s$ (Hudson 2002), allowing for a great range of demographic models and assumptions about recombination. While our proposed algorithm does not execute as quickly as the SMC algorithm (data not shown), it does support more demographic scenarios and allows for the incorporation of intragenic gene conversion. We illustrate how MaCS can be used in an applied context to simulate genome-wide case-control data under any specific disease model.

\section{Results}

\section{Implementation}

The algorithm for MaCS was implemented in C++ to maximize efficiency in terms of memory usage and speed while maintaining object-oriented design principles to facilitate future extensions. To make the program more widely applicable to human data, we implemented the same range of demographic models used in $m s$ (Hudson 2002), such as Wright's island model (Wright 1931). In general, these modifications were straightforward and were implemented in ways analogous to those used in the standard coalescent (Hudson 1991, 2002). We also included more subtle modeling capabilities, such as recombination rate variation using a piecewise constant model (Li and Stephens 2003) and intragenic gene conversion (Wiuf and Hein 2000). The mechanics behind these last two features are elaborated in the Methods section.

We first summarize the behavior of the algorithm behind MaCS, which is in essence a variation of the Wiuf and Hein algorithm (Wiuf and Hein 1999). To simulate a set of sequences, the Wiuf and Hein algorithm begins with a coalescent tree at the left end of the sequence, gradually constructing an ARG one recombination event at a time along the sequence until the full ARG is determined when the algorithm terminates at the right end of the sequence. Thus, the ARG at any point on the sequence captures only the recombination events that occurred among the set of sequences from that particular location to the left endpoint. Recombination events are assumed to follow a Poisson process along the sequence length so that the distance of each segment between recombination events is calculated from an exponential draw with an intensity parameter proportional to the scaled recombination rate $\rho$ and total ARG branch length $b$. In turn, $b$ is a function of the sample size $n$ and any demographic parameters. Mutations also occur as a Poisson process, similar to recombination events, except the scaled mutation rate $\theta$ replaces $\rho$. Specific details follow in Methods and are in the Wiuf and Hein article (Wiuf and Hein 1999). The entire sequence to be simulated is scaled to the unit interval [0,1], and time is scaled in units of $2 N_{e}$ generations, where $N_{e}$ is the diploid effective population size.
The Wiuf and Hein algorithm possesses the curious property that simulated recombination events become denser along the length of the sequence as the algorithm progresses further. As the current position moves to the right, the total branch length $b$ of the ARG increases, leading to shorter and shorter distances (i.e., shorter waiting times for the exponential draws) between successive recombination events. This happens because recombination can occur on any existing line of the ARG, including lines that are not ancestral to the set of sequences at the current position. One consequence is that the Wiuf and Hein approach is computationally infeasible for simulating large regions (e.g., whole chromosomes). In contrast, the more recent SMC algorithm (McVean and Cardin 2005) is much faster because only the last local tree (embedded within the ARG) is considered when (1) seeking the location of the next recombination event, and (2) determining which existing branch this new line eventually coalesces to. In our algorithm, we generalize the SMC by modifying step 2. In the SMC, coalescent events are restricted to edges within the last local tree only. In contrast, in our algorithm, at the point of recombination on the local tree, a new line emerges from an edge in the local tree and coalesces to an edge among any of the last $k$ (denoted as the tree-retention parameter in this article) local trees provided that the coalescent event occurs earlier in time than the recombination event and that the new line and the selected coalescing edge belong to the same population when simulating from an island model. Any edge that is unique to the local trees beyond (left of) the last $k$ local trees is pruned and not considered in the algorithm. A user-specified "history" parameter $h$ denotes a sequence length (in base pairs) that the algorithm uses to estimate how many local trees to retain as it simulates from the left end of the sequence to the right. Once the user specifies a sequence length $h$ (in base pairs), $k$ is then automatically computed based on the expected number of recombination events that would occur in a region of length $h$. Higher values of $h$ lead to better approximations of the standard coalescent, but at the cost of a slightly slower runtime and slightly greater memory requirements (see Performance section). For a given sample size $n$ and sequence length $h$, runtimes scale linearly with the simulated sequence length.

\section{Testing the accuracy of the method}

First, we compared the output of MaCS with the output from the standard coalescent simulator ms (Hudson 2002). Our interest was both in ensuring that our program was accurate and in determining whether storing more or less information (i.e., via the history parameter $h$ ) might bias estimates of coalescent times. We compared the times to the most recent common ancestor (TMRCA) averaged across 10 million replicates between MaCS and $m s$ for each of the first 15 trees and the final tree one encounters beginning at the left end of the sequence. Results are shown in Table 1 for $n=20$ sequences, using a scaled recombination rate parameter $\rho\left(=4 N_{e} L r\right)$ of 100 , which approximates an $L=167 \mathrm{~kb}$ region, assuming an effective population size $N_{e}=12,500$ and per-site recombination rate $r$ of $1.2 \times 10^{-8} / \mathrm{bp}$. In general, MaCS generates a distribution very similar to that of $m s$, even for the case of $h=1 \mathrm{bp}$ (equivalent to SMC). As $h$ increases (i.e., as we store more information about nearby genealogies), MaCS becomes a better approximation to $m s$. In the limit as $h \rightarrow \infty$ (when all previous local trees are stored), the average TMRCAs generated by MaCS are identical to those produced by $m s$, which reflects the fact that both algorithms are essentially simulating the same process. We compared the two programs 
Table 1. Average times to most recent common ancestor for 20 haplotypes across a $167-\mathrm{kb}$ region $(\rho=100)$

\begin{tabular}{ccccc}
\hline Tree & $\boldsymbol{m s}$ & $\boldsymbol{h}=\boldsymbol{\infty}^{\mathrm{a}}$ & $\boldsymbol{h}=\mathbf{1} \mathbf{~ k B}$ & $\boldsymbol{h = 1}$ \\
\hline 1 & 1.90 & 1.90 & 1.90 & 1.90 \\
2 & 1.95 & 1.96 & 1.96 & 1.96 \\
3 & 2.00 & 2.00 & 2.00 & 2.01 \\
4 & 2.03 & 2.04 & 2.04 & 2.05 \\
5 & 2.06 & 2.07 & 2.07 & 2.08 \\
6 & 2.08 & 2.09 & 2.09 & 2.10 \\
7 & 2.10 & 2.11 & 2.11 & 2.13 \\
8 & 2.12 & 2.12 & 2.13 & 2.15 \\
9 & 2.13 & 2.13 & 2.14 & 2.16 \\
10 & 2.14 & 2.14 & 2.15 & 2.18 \\
11 & 2.15 & 2.15 & 2.18 & 2.19 \\
12 & 2.16 & 2.16 & 2.18 & 2.20 \\
13 & 2.17 & 2.17 & 2.18 & 2.20 \\
14 & 2.18 & 2.17 & 2.19 & 2.21 \\
15 & 2.18 & 2.18 & 2.20 & 2.22 \\
last & 1.90 & 1.90 & 1.90 & 1.90 \\
\hline
\end{tabular}

One million trials were run, with standard errors $<10^{-3}$ for all trees. ${ }^{\mathrm{a}} h=\infty$ specifies that all simulated trees were retained.

using lower and higher values for the rate $\rho$ (e.g., $\rho=1$ and 1000). In all cases, behavior was consistent with the $\rho=100$ case, with essentially identical values when $h \rightarrow \infty$ and slightly inflated tree heights with lower values of $h$.

We also ran extensive simulations comparing sequence polymorphism data generated by the two programs. For a range of parameter values, we tabulated the mean and variance of simple summaries of the data, including $\theta_{W}$ (Watterson 1975), $\pi$ (Tajima 1983), $D^{\prime}$ (Lewontin 1964), and $r^{2}$ (Hill and Robertson 1968). In all cases, the distributions of summary statistics were indistinguishable between the two models (see Supplemental Table S1).

\section{Performance}

We now compare our program to other similar simulation programs in terms of computational time and memory requirements. For the standard coalescent, we compare with ms (Hudson 2002), which is substantially faster than other coalescent implementations (e.g., Laval and Excoffier 2004; Mailund et al. 2005) (data not shown). In addition to $m s$, we also include two other recently released programs: GENOME (Liang et al. 2007), which employs a retrospective approach to simulation similar to the coalescent, and FORWSIM (Padhukasahasram et al. 2008), which uses a forward-in-time simulation approach. To highlight the basic characteristics of the algorithms underlying all four implementations, we run simple test cases in which no popula- tion structure was specified. Table 2 presents running times and typical memory footprints for a range of different sample sizes and simulated sequence lengths. To ensure that all of the algorithms simulated data that were consistent with each other, we plotted LD decay curves across programs for each of the various scenarios. We did not discern any differences among the decay curves, suggesting that all four algorithms generated consistent LD patterns. We were not able to compare average tree heights as shown in Table 1 , since only output from MaCS and $m s$ provided us with the ability to determine tree heights.

We note that runtime speed for GENOME was similar to $m s$ for a small region, but substantially faster for the large region of $100 \mathrm{Mb}$. FORWSIM appears to use memory more efficiently than GENOME or $m s$. However, all three programs had substantial memory requirements, forcing several tests to be aborted early. Our program appears to scale well to large regions and samples sizes, and is generally both quicker and less memory intensive than the other tested programs.

\section{Applications}

As an example of how MaCS might be used, we simulated wholegenome SNP data meant to mimic the YRI data from the HapMap (Frazer et al. 2007). We first inferred phase in the YRI data using a hidden Markov model-based algorithm implemented in the program fastPHASE (Scheet and Stephens 2006). We then estimated demographic parameters for a two-population model using resequencing data from Yoruba and European samples in the Environmental Genome Project (Livingston et al. 2004) and a summary likelihood approach (Plagnol and Wall 2006). We estimated recent population growth $(20 \mathrm{kyr}$ ago $)$ in the Yoruba and a recent population bottleneck ( $20 \mathrm{kyr}$ ago) in the European population. Then, we simulated data under the best-fit model using MaCS and tabulated the decay of LD (measured by $r^{2}$ ) as a function of distance for nearby pairs of SNPs for the real and the simulated data. The results are shown in Figure 1. The curves for the simulated and actual data line up reasonably well, though it looks like there is less LD at short distances in the actual data. This is likely due to slight inaccuracies in the model assumptions, which could presumably be corrected if a more systematic study were undertaken (Schaffner et al. 2005).

We also note that the faster runtime of MaCS makes it more feasible to directly simulate case-control data under a specified disease model and prevalence. When evaluating novel methods for analyzing case-control data in GWAS, it is useful to not only assess how sensitive a method is (i.e., power), but also how well it performs with respect to the rate of false positives (i.e., specificity). While it is relatively easy to simulate scenarios to measure

Table 2. Average execution times and memory usage

\begin{tabular}{|c|c|c|c|c|c|c|}
\hline Region & Sample size & $m s$ & GENOME & FORWSIM & $\mathrm{MaCS} h=1 \mathrm{~Kb}$ & MaCS $h=1$ \\
\hline \multirow[t]{5}{*}{$10 \mathrm{Mb}$} & 100 & $5: 35$ (170 MB) & 4:19 (434 MB) & 0:05 (99 MB) & $0: 13$ (9.6 MB) & $0: 11$ (9.6 MB) \\
\hline & 300 & 8:08 (308 MB) & $5: 05(1.1 \mathrm{~GB})$ & $1: 22(153 \mathrm{MB})$ & $0: 43(10 \mathrm{MB})$ & $0: 41(10 \mathrm{MB})$ \\
\hline & 1000 & $10: 47$ (1.1 GB) & $6: 18(3.8 \mathrm{~GB})$ & $37: 36$ (582 MB) & $3: 00$ (11.7 MB) & $2: 42(11.7 \mathrm{MB})$ \\
\hline & 3000 & $19: 50$ (3.6 GB) & $18: 13(16.9 \mathrm{~GB})$ & $12 \mathrm{~h}(3.8 \mathrm{~GB})$ & $13: 05$ (13.8 MB) & $9: 31$ (13.8 MB) \\
\hline & 10,000 & 1:08:11 (13.3 GB) & $\mathrm{N} / \mathrm{A}(>18 \mathrm{~GB})$ & $\mathrm{N} / \mathrm{A}(>18 \mathrm{~GB})$ & $50: 50$ (20.4 MB) & $47: 50(20.2 \mathrm{MB})$ \\
\hline \multirow[t]{5}{*}{$100 \mathrm{Mb}$} & 100 & $13 \mathrm{~d}(942 \mathrm{MB})$ & $51: 38(4.2 \mathrm{~GB})$ & $2: 31(820 \mathrm{MB})$ & $2: 15(28 \mathrm{MB})$ & $2: 09(28 \mathrm{MB})$ \\
\hline & 300 & $>1 \mathrm{mo}(>3 \mathrm{~GB})$ & $58: 31$ (8.5 GB) & $53: 54$ (1.1 GB) & $7: 19$ (36 MB) & $6: 36$ (36 MB) \\
\hline & 1000 & $\mathrm{~N} / \mathrm{A}(>18 \mathrm{~GB})$ & $\mathrm{N} / \mathrm{A}(>18 \mathrm{~GB})$ & $29 \mathrm{~h}(4.7 \mathrm{~GB})$ & $24: 03(40.7 \mathrm{MB})$ & $21: 40(40.5 \mathrm{MB})$ \\
\hline & 3000 & $\mathrm{~N} / \mathrm{A}(>18 \mathrm{~GB})$ & $\mathrm{N} / \mathrm{A}(>18 \mathrm{~GB})$ & $\mathrm{N} / \mathrm{A}(>18 \mathrm{~GB})$ & $2: 21: 24(45.8 \mathrm{MB})$ & $1: 54: 45(44.9 \mathrm{MB})$ \\
\hline & 10,000 & $\mathrm{~N} / \mathrm{A}(>18 \mathrm{~GB})$ & $\mathrm{N} / \mathrm{A}(>18 \mathrm{~GB})$ & N/A (>18 GB) & $9 \mathrm{~h}(56 \mathrm{MB})$ & $9 \mathrm{~h}(56 \mathrm{MB})$ \\
\hline
\end{tabular}

Memory usage in parentheses. N/A entries denote test cases that were terminated because requested RAM exceeded the system's resources.

\section{Genome Research}

www.genome.org 


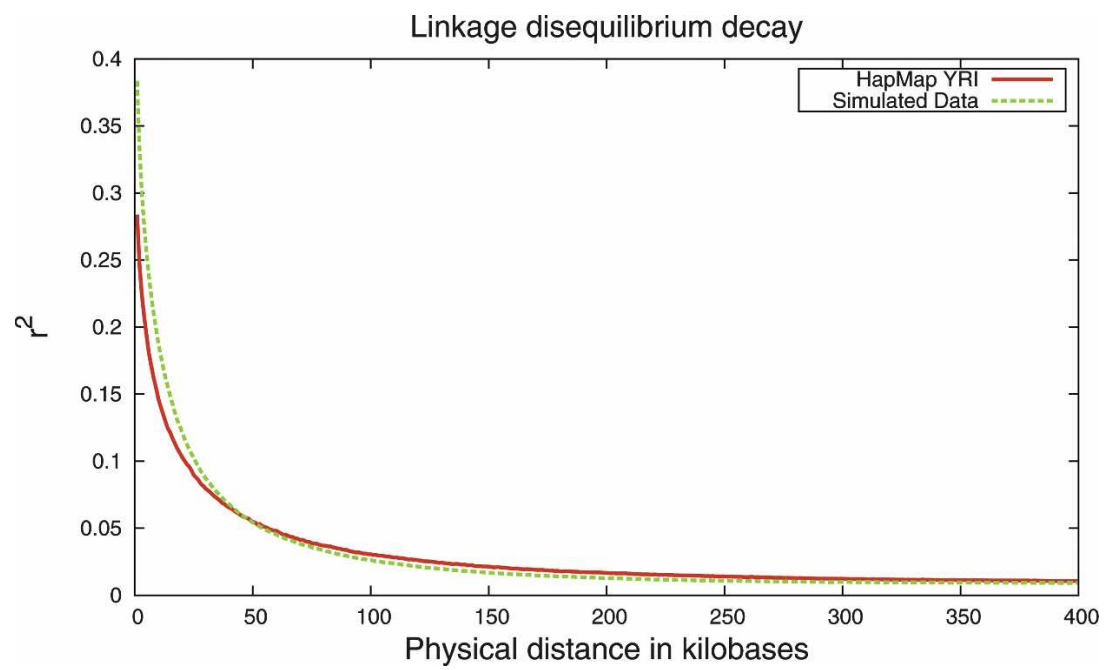

Figure 1. Average $r^{2}$ values were computed across all pairs of nearby (maximum $1 \mathrm{Mb}$ ) SNPs for a $270-\mathrm{Mb}$ region. Values for simulated data and HapMap YRI (Chromosome 1) are indicated by the green and red lines, respectively.

power, estimating specificity is generally more difficult, requiring a realistic representation of the null distribution. Because our program is model based, a variety of demographic models can be tested, an advantage over schemes that either permute casecontrol labels on existing data or perturb existing genetic data as described earlier in this text.

We tested the computational feasibility of simulating casecontrol data using our method assuming a model based on data from Type 2 diabetes studies. The transcription factor-encoding gene TCF7L2 has been widely implicated in disease risk across several independent studies (Cauchi and Froguel 2008). Here we assume a population prevalence $K$ of $7 \%$ for Type 2 diabetes, a minor allele frequency of TCFL2 of $26 \%$, and heterozygous (RR1) and mutant homozygous relative risks (RR2) of 1.45 and 2.41 (Grant et al. 2006). To obtain 2000 cases, we calculated the sample size needed to be 33,333 , or $\sim 67,000$ haplotypes. Rounding up, we simulated 100,000 haplotypes for a $10-\mathrm{Mb}$ region. This took 983 min to complete, requiring $93 \mathrm{MB}$ of RAM. Because runtime is linear in sequence length, we can extrapolate that simulating 100,000 copies of Chromosome $1(\sim 270 \mathrm{Mb})$, should require $\sim 40 \mathrm{~h}$ and $270 \mathrm{MB}$ of RAM. Once data are generated, it is straightforward to produce a SNP panel similar to a genotype chip by ascertaining common SNPs (as described in Methods) and to mask a random SNP with the desired disease allele frequency.

\section{Discussion}

Simulation-based analyses of DNA sequence data have a long and fruitful history in evolutionary and human genetics. They are used to estimate evolutionary parameters, distinguish between demographic models, detect the effects of natural selection, and test the accuracy of fine-scale mapping methods. As molecular sequencing technology improves, computer simulations will be used in these and many other scenarios to help analyze increasingly large data sets-whole genome sequence polymorphism data sets are already available (Begun et al. 2007; Clark et al. 2007) and will soon become commonplace. We developed MaCS in response to these developments. Whole chromosome simula- tions can be performed in minutes to hours on a standard desktop computer (Table 2), much faster than any other simulation program. Although our algorithm loses efficiency in terms of speed as sample size is increased, we have shown that it is possible to simulate the number of samples necessary for studying common diseases. We have also designed MaCS to allow users to specify the level of accuracy they desire in the approximation of the coalescent through the command line parameter $h$. Setting $h$ to its minimum value of $1 \mathrm{bp}$ can still provide a fairly good approximation to the coalescent (Table 1; Marjoram and Wall 2006). In general, however, we recommend setting $h$ to larger values, especially when the user wishes to accommodate gene conversions, in which case $h$ should be longer than typical gene conversion tract lengths. Setting $h$ to $1 \mathrm{~kb}$, well beyond the assumed lengths of gene conversion tracts in humans, we have found that our algorithm does not suffer any substantive effect in terms of performance (Table 2). It is our hope that MaCS will help facilitate the analyses of large-scale data sets in the same way that standard coalescent simulation programs (Hudson 1991, 2002) have done for single-locus DNA sequence data sets.

\section{Methods}

Our modified Wiuf and Hein algorithm (Wiuf and Hein 1999) can be outlined as follows:

1. Initialize the local tree ID variable $i$ as 0 . At position $x_{\text {cur }}=0$ (the left endpoint of the sequence), a coalescent tree $T_{o}$ based on the present day set of sequences is constructed using standard coalescent methodology (Kingman 1982; Hudson 1991), where each edge is labeled with the value $i$. The full data structure is a graph, denoted as $G$. At this point, $G=T_{O}$. Once the tree is constructed, increment $i$ by 1 .

2. Save the current position $x_{\text {cur }}$ along the sequence as $x_{\text {prev }}$.

3. The distance $x_{\mathrm{r}}$ to the first recombination point along the chromosome is taken from an exponential draw $x_{\mathrm{r}} \sim \exp (b \rho)$, where the rate is a function of the total branch length $b$ of the current local tree $T_{i}$ and the scaled recombination rate $\rho$ ( $=4 \mathrm{Nr}$, where $r$ is the per-generation recombination rate scaled across the length of sequence).

4. The distance $x_{\mathrm{m}}$ to the first mutation point along the chromosome is taken from an exponential draw $x_{\mathrm{m}} \sim \exp (b \theta)$, where $b$ is defined as in step 3, and mutation rate $\theta$ is defined as $4 N \mu, \mu$ being the per-generation mutation rate scaled across the length of sequence.

5. Update the current position along the sequence, defined as $x_{\text {cur }}=x_{\text {prev }}+x_{\mathrm{m}}$.

6. If $x_{\text {cur }}<\left(x_{\text {prev }}+x_{\mathrm{r}}\right)$, randomly choose a mutation site uniformly on the interval $[0, b]$ within $T_{i}$. Any present day samples that are descendants of this point contain the mutation. Loop through all samples, printing 1 for the samples with mutations and 0 for the others.

7. Repeat steps 4-6 until $x_{\text {cur }}>x_{\text {prev }}+x_{\mathrm{r}}$, at which point set $x_{\text {cur }}=x_{\text {prev }}+x_{\mathrm{r}}$ using the memory-less property of the exponential distribution. 
8. Randomly choose a recombination site on the interval $[0, b]$ within $T_{i}$

9. A new lineage is extended backward in time from the recombination point by forking off the recombination node and finding a new edge in $G$ to coalesce to. This new line, which is labeled with the value $i$, can coalesce with any existing lines of ancestry by merging with the existing line at an earlier point in time (i.e., higher in the graph than the recombination point). If there are $l$ remaining lines of ancestry, then the new lineage coalesces at rate $l$ with a randomly chosen line. Thus, the waiting time $t$ before coalescence is exponentially distributed $t \sim \exp (l)$. Note that $l$ changes over time according to prespecified coalescent and recombination events since the number of existing edges changes as one moves up the graph. Traverse all edges that descend from the recombination point, relabeling each of these edges with the value $i$.

10. Define the new local tree $T_{i}$ within $G$ by labeling appropriate edges in $G$ as follows: Mark edges with the current local tree ID $i$ by traversing the edges of $G$ upward from the present time for each sample until a common ancestor is found given the two constraints:

(a) The putative common ancestor cannot be lower than any point on any edge in $G$ labeled with the value $i$.

(b) When traversing up edges and a recombination node is encountered (where two edges emerge above it), choose the edge with the larger tree ID value.

11. Prune any edge with tree ID less than or equal to $i-k$, where $k$ is the tree-retention parameter described earlier.

12. Increment the tree ID variable $i$ by 1 .

13. Repeat steps 2-12 until the right endpoint (i.e., $x_{\text {prev }}+x_{\mathrm{r}}>1.0$ ) of the sequenced region is reached. Replace $b$ in step 3 with $b_{r}$, the total branch length of the ARG consisting of the last $k$ local trees.

14. The algorithm is now complete. The output from step 5 across the entire region constitutes the sequence data, where 0 and 1 represent the ancestral and derived alleles, respectively.

By labeling edges within a graph, we can store a set of local trees more efficiently than if we were to explicitly store the local trees separately as required by Hudson's algorithm (Hudson 2002). However, this large improvement in memory efficiency is gained at the expense of some efficiency in speed. By storing information in such a data structure, the number of physical edges that must be traversed to determine a local tree is often greater than the theoretical value of $n(n-1) / 2$, since edges can be fragmented by nodes marking migration events (under advanced demographic scenarios) or recombination nodes retained from previous local trees. Due to this fragmentation, the algorithm becomes less efficient, particularly at the step where edge labels need to be updated to determine the next local tree.

The behavior of our algorithm is illustrated in Figure 2 with a concrete example in which three sequences are simulated with the "tree-retention" parameter $k$ fixed at 2 . The set of thick dashed lines at the bottom of the figure

\section{Left Endpoint}

is an ideogram of the sequences to be simulated, and the graphs above the dashed lines indicate the topology of the current state of the graph at each particular step in our algorithm. We consider the vertical line immediately above each sequence to be a lineage. For brevity, we present the graph topologies for only the first two recombination events, along with a pruning event. Here, each graph vertex (node) with at least two edges represents either a recombination or coalescent event. Each vertical edge is labeled according to the identity of the most recent local tree it was a part of. Since horizontal edges carry no information regarding time between events, they are not labeled in the figure.

1. The first topology shows the graph initialized as a coalescent tree $T_{O}$ at the start of the algorithm.

2. The first recombination event occurs at the point along the sequence indicated by the second topology. The new coalescing line is labeled with the identity of the new local tree ID (i.e., 1) as well as any lines that are descendants of the recombination point.

3. A new local tree $T_{1}$ shown in bold lines is subsequently defined in the third topology by marking the edges to the value 1 according to the marking algorithm described above.

4. The fourth topology shows the second recombination event followed by a coalescent event above the MRCA. The same rules described in step 2 apply here.

5. The fifth topology repeats the marking algorithm described earlier, with edges labeled 2.

6. A pruning event is invoked since $k=2$ and the current tree ID $i$ is equal to 2 . Thus, any edges with tree IDs less than or equal to 0 will be pruned from the graph.

Steps 2-6 may be repeated hundreds of thousands of times when simulating long chromosomes for large sample sizes.

\section{Variable recombination rates}

A set of mappings between the unit interval $[0,1]$ and recombination rate ratio $(\mathrm{cM} / \mathrm{Mb})$ mappings can be provided to MaCS through a flat file. For example, if a 1-MB region is to be simulated, and the first $100 \mathrm{kB}$ is expected to have twice the baseline recombination rate across the entire sequence, the first

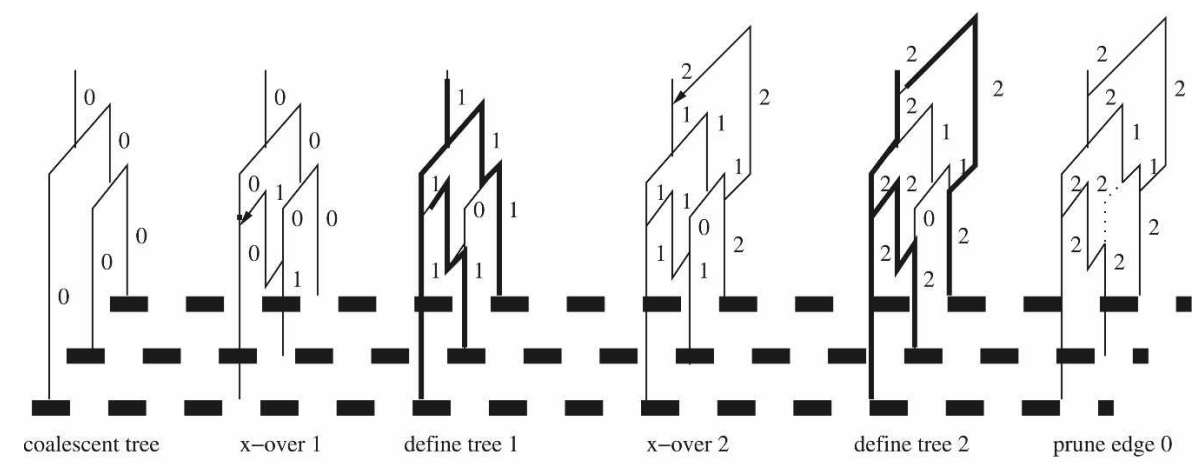

Figure 2. A demonstration of the algorithm behind MaCS for a sample of three sequences and the tree-retention parameter set to $k=2$. The algorithm proceeds from the left end of the region to be simulated toward the right end. Vertical edges are labeled to their immediate right with the ID of the most recent tree that it belongs to.

\section{Genome Research}

www.genome.org 
line in the input file can be read into the program as $0<\mathrm{tab}>0.10<\mathrm{tab}>2.0$.

The family of algorithms derived from the Wiuf and Hein method (Wiuf and Hein 2000), including SMC and MaCS, can easily accommodate recombination rate variation. At any userspecified zone where the rate ratio is expected to deviate from 1 , the $b \rho$ parameter in the exponential draw for the next recombination point is scaled by the rate ratio.

\section{Gene conversion}

From a topological standpoint, simple crossovers can be modeled within the graph as a recombining node with two lines emerging above it. One line, the "old" line, represents the original line leading to its ancestors to the left of the recombination event; the other line, the "new" line, leads to its ancestors to the right of the recombination event. Gene conversion can be modeled within a graph as a crossover event, immediately followed by a second crossover event (close to the first crossover), with the added condition that the new line of the second crossover event coalesces to the old line of the first event, effectively forming a loop in the graph. This concept is illustrated clearly by Wiuf and Hein (2000).

We implement gene conversion using a model that incorporates a parameter $f$ that reflects the ratio of the rate of gene conversion events and the rate of crossover events (Frisse et al. 2001) and then assumes a geometric distribution of gene conversion tract lengths $\mathrm{L}$ :

$$
\operatorname{Pr}(L=n)=(1-\varphi) \varphi^{\mathrm{n}}
$$

where $n$ is the number of nucleotides and $\varphi$ is the probability a tract continues once it begins (Hilliker et al. 1994). Estimates of $\varphi$, which determine the expected tract length, vary among organisms, and its estimation is beyond the scope of this paper. By use of the same general algorithm as described above, we increase the recombination rate at any position by a factor of $(1+f)$. Each recombination event is then labeled as a crossover with probability $1 /(1+f)$ or a gene conversion event with probability $f /(1+f)$.

If the next event is a gene conversion event, we generate the tract length and add to the graph the first crossover event of the gene conversion. The recombining node and the endpoint (scaled on the unit interval) for the tract is then stored in memory until the algorithm reaches the endpoint of the tract. At this point, we recall the two edges emerging upward from the recombining node and add the second crossover event to the graph, thus completing the gene conversion loop. Note that there may be several gene conversion tracts that overlap one another (especially with high $f$ values). In certain cases, the gene conversion loop cannot be completed. For instance, the old line can be deleted if the edge label of this line is beyond the threshold dictated by the tree-retention parameter $k$. Another case is when a tract begins near the end of the simulated sequence and the tract length endpoint exceeds the end $(>1)$ of the total sequence.

\section{Ascertainment of segregating sites}

When simulating scenarios to model data generated by commercial genotyping products, one needs to pay attention to the schemes used to ascertain the markers. Because commercial products are usually geared toward optimizing statistical power, markers with rare minor alleles are almost always excluded. Most simulation programs available, including $m s$, have provisions to exclude sites with derived allele frequency less than a user- specified threshold. We offer a more general approach by allowing the user to provide an input file containing a list of bins (identified as a range of minor allele frequencies) along with the frequencies of mutations within each bin. MaCS will then produce simulations that mimic this distribution of allele frequencies. For example, in the specific case where one would like to filter out any SNPs $<1 \%$, one would include one line in the input file that reads $0<\operatorname{tab}>0.01<\operatorname{tab}>0.0$, which informs the program that any SNPs with a derived allele frequency between 0 and 0.01 has a probability 0 of being output. Whenever feasible, we do this by simulating as described before but with a higher mutation rate and then iteratively thinning the data until the simulated frequency spectrum matches the specified distribution of allele frequencies. However, in cases where the user-specified constraints cannot be met (e.g., insufficient number of segregating sites to remove), we notify the user and do not post-process the data as described above. Note that this approach is equivalent to the ascertainment correction scheme described by Voight and colleagues (2006).

\section{Benchmarking across programs}

Generating comparable results from four different programs requires some careful planning since each program interprets parameters differently. Specifically, the parameters $N_{e}$ (effective population size), $\mu$ (per site mutation rate), and $r$ (per site recombination rate) should be common across all test cases. Here, we assumed $N_{e}=12,500, \mu=2 \times 10^{-8} / \mathrm{bp}$, and $r=1.2 \times 10^{-8} / \mathrm{bp}$. This translates to a scaled mutation rate and recombination rate (scaled in units of $4 N_{e}$ generations) of 10,000 and 6000 for a $10-\mathrm{Mb}$ region, values that are directly provided to the $m s$ command line. We should expect to observe similar mutation and recombination counts since all four algorithms assume that mutations and recombination events follow a Poisson process with intensity dictated only by the total branch length across sites and the scaled mutation/recombination rates.

A distinct option of GENOME is the ability for the user to specify a minimum tract length, $d$, of DNA in which no recombination occurs. Consequently, if the length of the region being simulated is $L$, then the maximum number of recombination events one can expect to observe is given by $L /(d-1)$. The documentation notes that the tract length can be set to a 1 (i.e., a single base pair) when sampling small regions but, for the sake of memory efficiency, should be increased. To estimate what value might be appropriate, we first ran simulations using $m s$ for each of the scenarios in Table 2 to obtain a rough estimate of the expected number of trees (recombination events) and mutations (segregating sites). We used the number of distinct trees generated by $m s$ to derive the minimum tract length value in GENOME, and thus an upper bound for the number of recombination events. Note that imposing this hard constraint is likely to lead to a bias toward a lower than expected number of trees, but we felt that given the heavy memory burden, this was a small cost.

For FORWSIM simulations, we set $\mu$ and $r$ as above, but allow $N_{e}$ to vary across different runs. Specifically, we define $N_{e}=10 n$ (where $n$ is the sample size), and follow each simulation for $10 N_{e}$ generations. For forward-time simulations to be accurate, the population size must be large enough for the scaling to be appropriate, but simulating large population sizes is costly both in terms of runtime and memory usage. Our approach is something of a compromise, and we have not tested specifically whether the $N_{e}$ values chosen are large enough (i.e., whether FORWSIM simulations are good approximations of the neutral evolutionary process for the particular values chosen).

To better gauge the baseline memory overhead across algo- 
rithms, we polled memory usage at 1-sec intervals and calculated the maximum for each test run, as indicated in Table 2.

\section{Estimating demographic parameters for Yoruba HapMap data}

We used the method described previously (Plagnol and Wall 2006) to estimate demographic parameters appropriate for the Yoruba HapMap data. Briefly, we used data from the NIEHS SNPs project (Livingston et al. 2004) to estimate demographic parameters under a two-population model meant to mimic the history of West African and European populations. This model incorporated divergence and migration between populations, recent population growth, and a population bottleneck in one of the populations. We summarized the data using a variety of summaries of the joint frequency spectrum and then used coalescent simulations to determine demographic parameters that maximized the likelihood of the data. For further details, see Plagnol and Wall (2006).

\section{Estimation of sample sizes necessary for case-control simulation}

In the example for Type 2 diabetes, we can solve for the penetrance probabilities $f_{0}, f_{1}$, and $f_{2}$ for the wild-type homozygote, heterozygote, and mutant homozygote genotype groups, respectively, given a risk allele frequency $p_{\mathrm{d}}$ using the relationship:

$$
K=\left(1-p_{\mathrm{d}}\right)^{2} f_{\mathrm{o}}+2 p_{\mathrm{d}}\left(1-p_{\mathrm{d}}\right) f_{1}+p_{\mathrm{d}}^{2} f_{2}
$$

where

$$
\operatorname{RR} 1=f_{1} / f_{\mathrm{o}} \text { and } \mathrm{RR} 2=f_{2} / f_{\mathrm{o}} .
$$

Given the heterozygote (RR1) and homozygote (RR2) relative risks, in order to calculate the sample size $N$ required to have expected $n$ cases, we can substitute the values above into:

$$
n=N\left(p_{\mathrm{d}}^{2} f_{2}+2 p_{\mathrm{d}}\left(1-p_{\mathrm{d}}\right) f_{1}+\left(1-p_{\mathrm{d}}\right)^{2} f_{\mathrm{o}}\right) .
$$

\section{Acknowledgments}

This research was funded by National Institutes of Health grants HG004049 (P.M and J.D.W) and R25T CA112355 (G.K.C. Fellow).

\section{References}

Begun, D.J., Holloway, A.K., Stevens, K., Hillier, L.W., Poh, Y.P., Hahn, M.W., Nista, P.M., Jones, C.D., Kern, A.D., Dewey, C.N., et al. 2007. Population genomics: Whole-genome analysis of polymorphism and divergence in Drosophila simulans. PLoS Biol. 5: e310. doi: 10.1371/journal.pbio.0050310.

Cauchi, S. and Froguel, P. 2008. TCF7L2 genetic defect and type 2 diabetes. Curr. Diab. Rep. 8: 149-155.

Clark, R.M., Schweikert, G., Toomajian, C., Ossowski, S., Zeller, G., Shinn, P., Warthmann, N., Hu, T.T., Fu, G., Hinds, D.A., et al. 2007. Common sequence polymorphisms shaping genetic diversity in Arabidopsis thaliana. Science 317: 338-342.

Durrant, C., Zondervan, K.T., Cardon, L.R., Hunt, S., Deloukas, P., and Morris, A.P. 2004. Linkage disequilibrium mapping via cladistic analysis of single-nucleotide polymorphism haplotypes. Am. J. Hum. Genet. 75: 35-43.

Frazer, K.A., Ballinger, D.G., Cox, D.R., Hinds, D.A., Stuve, L.L., Gibbs, R.A., Belmont, J.W., Boudreau, A., Hardenbol, P., Leal, S.M., et al. 2007. A second generation human haplotype map of over 3.1 million SNPs. Nature 449: 851-861.

Frisse, L., Hudson, R.R., Bartoszewicz, A., Wall, J.D., Donfack, J., and Di Rienzo, A. 2001. Gene conversion and different population histories may explain the contrast between polymorphism and linkage disequilibrium levels. Am. J. Hum. Genet. 69: 831-843.

Grant, S.F., Thorleifsson, G., Reynisdottir, I., Benediktsson, R., Manolescu, A., Sainz, J., Helgason, A., Stefansson, H., Emilsson, V., Helgadottir, A., et al. 2006. Variant of transcription factor 7-like 2 (TCF7L2) gene confers risk of type 2 diabetes. Nat. Genet. 38:
$320-323$.

Griffiths, R.C. and Marjoram, P. 1996. Ancestral inference from samples of DNA sequences with recombination. J. Comput. Biol. 3: 479-502.

Hill, W.G. and Robertson, A. 1968. Linkage disequilibrium in finite populations. Theor. Appl. Genet. 38: 226-231.

Hilliker, A.J., Harauz, G., Reaume, A.G., Gray, M., Clark, S.H., and Chovnick, A. 1994. Meiotic gene conversion tract length distribution within the rosy locus of Drosophila melanogaster. Genetics 137: 1019-1026.

Hudson, R.R. 1991. Gene geneologies and the coalescent process. In Oxford surveys in evolutionary biology (eds. D. Futuyama and J. Antonovics), pp. 1-44. Oxford University Press, UK.

Hudson, R.R. 2002. Generating samples under a Wright-Fisher neutral model of genetic variation. Bioinformatics 18: 337-338.

Kingman, J.F.C. 1982. The coalescent. Stochastic Process. Appl. 13: $235-248$.

Laval, G. and Excoffier, L. 2004. SIMCOAL 2.0: A program to simulate genomic diversity over large recombining regions in a subdivided population with a complex history. Bioinformatics 20: 2485-2487.

Lewontin, R.C. 1964. The interaction of selection and linkage. I. General considerations; heterotic models. Genetics 49: 49-67.

Li, N. and Stephens, M. 2003. Modeling linkage disequilibrium and identifying recombination hotspots using single-nucleotide polymorphism data. Genetics 165: 2213-2233.

Liang, L., Zollner, S., and Abecasis, G.R. 2007. GENOME: A rapid coalescent-based whole genome simulator. Bioinformatics 23: 1565-1567.

Livingston, R.J., von Niederhausern, A., Jegga, A.G., Crawford, D.C., Carlson, C.S., Rieder, M.J., Gowrisankar, S., Aronow, B.J., Weiss, R.B., and Nickerson, D.A. 2004. Pattern of sequence variation across 213 environmental response genes. Genome Res. 14: 1821-1831.

Mailund, T., Schierup, M.H., Pedersen, C.N., Mechlenborg, P.J., Madsen, J.N., and Schauser, L. 2005. CoaSim: A flexible environment for simulating genetic data under coalescent models. BMC Bioinformatics 6: 252. doi: 10.1186/1471-2105-6-252.

Marjoram, P. and Wall, J.D. 2006. Fast "coalescent" simulation. BMC Genet. 7: 16. doi: 10.1186/1471-2156-7-16.

McVean, G.A. and Cardin, N.J. 2005. Approximating the coalescent with recombination. Philos. Trans. R. Soc. Lond. B Biol. Sci. 360: 1387-1393.

Padhukasahasram, B., Marjoram, P., Wall, J.D., Bustamante, C.D., and Nordborg, M. 2008. Exploring population genetic models with recombination using efficient forward-time simulations. Genetics 178: $2417-2427$.

Plagnol, V. and Wall, J.D. 2006. Possible ancestral structure in human populations. PLoS Genet. 2: e105. doi: 10.1371/journal.pgen.0020105.

Reich, D.E., Cargill, M., Bolk, S., Ireland, J., Sabeti, P.C., Richter, D.J., Lavery, T., Kouyoumjian, R., Farhadian, S.F., Ward, R., et al. 2001. Linkage disequilibrium in the human genome. Nature 411: 199-204.

Schaffner, S.F., Foo, C., Gabriel, S., Reich, D., Daly, M.J., and Altshuler, D. 2005. Calibrating a coalescent simulation of human genome sequence variation. Genome Res. 15: 1576-1583.

Scheet, P. and Stephens, M. 2006. A fast and flexible statistical model for large-scale population genotype data: Applications to inferring missing genotypes and haplotypic phase. Am. J. Hum. Genet. 78: 629-644.

Stram, D.O. 2004. Tag SNP selection for association studies. Genet. Epidemiol. 27: 365-374.

Tajima, F. 1983. Evolutionary relationship of DNA sequences in finite populations. Genetics 105: 437-460.

Voight, B.F., Adams, A.M., Frisse, L.A., Qian, Y., Hudson, R.R., and Di Rienzo, A. 2005. Interrogating multiple aspects of variation in a full resequencing data set to infer human population size changes. Proc. Natl. Acad. Sci. 102: 18508-18513.

Voight, B.F., Kudaravalli, S., Wen, X., and Pritchard, J.K. 2006. A map of recent positive selection in the human genome. PLoS Biol. 4: e72.doi: 10.1371/journal.pbio.0040072.

Wall, J.D. 2004. Close look at gene conversion hot spots. Nat. Genet. 36: $114-115$.

Watterson, G.A. 1975. On the number of segregating sites in genetical models without recombination. Theor. Popul. Biol. 7: 256-276.

Wiuf, C. and Hein, J. 1999. Recombination as a point process along sequences. Theor. Popul. Biol. 55: 248-259.

Wiuf, C. and Hein, J. 2000. The coalescent with gene conversion. Genetics 155: 451-462.

Wright, S. 1931. Evolution in Mendelian populations. Genetics 16: 97-159.

Received July 22, 2008; accepted in revised form October 7, 2008.

\section{Genome Research}

www.genome.org 


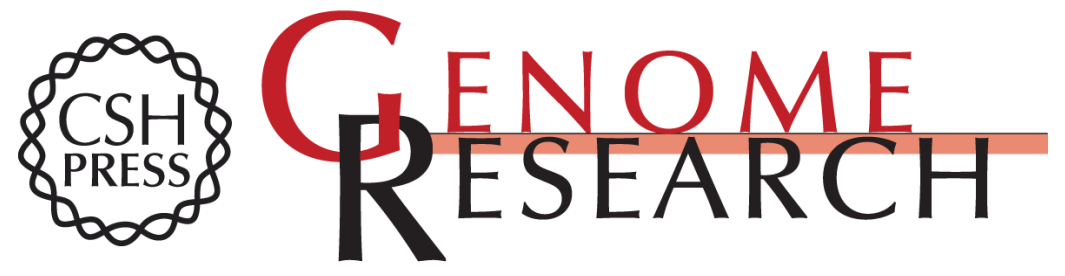

\section{Fast and flexible simulation of DNA sequence data}

Gary K. Chen, Paul Marjoram and Jeffrey D. Wall

Genome Res. 2009 19: 136-142 originally published online November 24, 2008

Access the most recent version at doi:10.1101/gr.083634.108

Supplemental http://genome.cshlp.org/content/suppl/2008/11/25/gr.083634.108.DC1
Material

References This article cites 34 articles, 11 of which can be accessed free at:

http://genome.cshlp.org/content/19/1/136.full.html\#ref-list-1

\section{License}

Email Alerting Receive free email alerts when new articles cite this article - sign up in the box at the Service top right corner of the article or click here.

\section{Affordable, Accurate Sequencing.}

